\title{
Characterizations of centralizable mappings on algebras of locally measurable operators
}

\author{
Guangyu $\mathrm{An}^{1}$, Jun $\mathrm{He}^{2 *}$ and Jiankui $\mathrm{Li}^{3}$ \\ ${ }^{1}$ Department of Mathematics, Shaanxi University of Science and Technology \\ Xi'an 710021, China \\ ${ }^{2}$ Department of Mathematics, Anhui Polytechnic University \\ Wuhu 241000, China \\ ${ }^{3}$ Department of Mathematics, East China University of Science and Technology \\ Shanghai 200237, China
}

\begin{abstract}
A linear mapping $\phi$ from an algebra $\mathcal{A}$ into its bimodule $\mathcal{M}$ is called a centralizable mapping at $G \in \mathcal{A}$ if $\phi(A B)=\phi(A) B=A \phi(B)$ for each $A$ and $B$ in $\mathcal{A}$ with $A B=G$. In this paper, we prove that if $\mathcal{M}$ is a von Neumann algebra without direct summands of type $\mathrm{I}_{1}$ and type II, $\mathcal{A}$ is a *-subalgebra with $\mathcal{M} \subseteq \mathcal{A} \subseteq L S(\mathcal{M})$ and $G$ is a fixed element in $\mathcal{A}$, then every continuous (with respect to the local measure topology $t(\mathcal{M})$ ) centralizable mapping at $G$ from $\mathcal{A}$ into $\mathcal{M}$ is a centralizer.
\end{abstract}

Keywords: centralizable mapping, centralizer, von Neumann algebra, locally measurable operator

Mathematics Subject Classification(2010):46L57; 47L35; 46L50

\section{Introduction}

Let $\mathcal{A}$ be an associative algebra over the complex field $\mathbb{C}, \mathcal{M}$ be an $\mathcal{A}$-bimodule, and $L(\mathcal{A}, \mathcal{M})$ be the set of all linear mappings from $\mathcal{A}$ into $\mathcal{M}$. If $\mathcal{A}=\mathcal{M}$, then denote $L(\mathcal{A}, \mathcal{A})$ by $L(\mathcal{A})$. A linear mapping $\phi$ in $L(\mathcal{A}, \mathcal{M})$ is called a centralizer if

\footnotetext{
${ }^{*}$ Corresponding author. E-mail address: hejun_12@163.com
} 
$\phi(A B)=\phi(A) B=A \phi(B)$ for each $A$ and $B$ in $\mathcal{A}$. In particular, if $\mathcal{A}$ is a unital algebra with a unit element $I$, then $\phi$ is a centralizer if and only if $\phi(A)=\phi(I) A=A \phi(I)$ for every $A$ in $\mathcal{A}$.

Let $G$ be a fixed element in $\mathcal{A}$. A linear mapping $\phi$ in $L(\mathcal{A}, \mathcal{M})$ is called a centralizable mapping at $G$ if $\phi(A B)=\phi(A) B=A \phi(B)$ for each $A$ and $B$ in $\mathcal{A}$ with $A B=G$. Moreover, we say that $G$ is a full-centralizable point of $L(\mathcal{A}, \mathcal{M})$ if every centralizable mapping at $G$ from $\mathcal{A}$ into $\mathcal{M}$ is a centralizer.

Suppose that $\mathcal{R}$ is a prime ring with a nontrival idempotent, in [5], M. Brešar shows that zero is a full-centralizable point of $L(\mathcal{R})$; and in [13, X. Qi shows that every nontrival idempotent in $\mathcal{R}$ is a full-centralizable point of $L(\mathcal{R})$. In [16, W. Xu, R. An and J. Hou prove that if $\mathcal{H}$ is a Hilbert space with $\operatorname{dim} \mathcal{H} \geq 2$, then every element $G$ in $B(\mathcal{H})$ is a full-centralizable point of $L(B(\mathcal{H}))$. In [8], J. He, J. Li and W. Qian prove that if $\mathcal{M}$ is a von Neumann algebra, then every element $G$ in $\mathcal{M}$ is a full-centralizable point of $L(\mathcal{M})$.

Let $\mathcal{H}$ be a complex Hilbert space and $B(\mathcal{H})$ be the algebra of all bounded linear operators on $\mathcal{H}$. Suppose that $\mathcal{M}$ is a von Neumann algebra on $\mathcal{H}$ and $\mathcal{Z}(\mathcal{M})=\mathcal{M} \cap \mathcal{M}^{\prime}$ is the center of $\mathcal{M}$, where $\mathcal{M}^{\prime}=\{A \in B(\mathcal{H}): A B=B A$ for every $B$ in $\mathcal{M}\}$. Denote by $\mathcal{P}(\mathcal{M})=\left\{P \in \mathcal{M}: P=P^{*}=P^{2}\right\}$ the lattice of all projections in $\mathcal{M}$ and by $\mathcal{P}_{\text {fin }}(\mathcal{M})$ the set of all finite projections in $\mathcal{M}$.

Let $T$ be a closed densely defined linear operator on $\mathcal{H}$ with the domain $\mathcal{D}(T)$, where $\mathcal{D}(T)$ is a linear subspace of $\mathcal{H}$. $T$ is said to be affiliated with $\mathcal{M}$, denote by $T \eta \mathcal{M}$, if $U^{*} T U=T$ for every unitary element $U$ in $\mathcal{M}^{\prime}$.

A linear operator $T$ affiliated with $\mathcal{M}$ is said to be measurable with respect to $\mathcal{M}$, if there exists a sequence $\left\{P_{n}\right\}_{n=1}^{\infty} \subset \mathcal{P}(\mathcal{M})$ such that $P_{n} \uparrow 1, P_{n}(\mathcal{H}) \subset \mathcal{D}(T)$ and $P_{n}^{\perp}=I-P_{n} \in \mathcal{P}_{\text {fin }}(\mathcal{M})$ for every $n \in \mathbb{N}$, where $\mathbb{N}$ is the set of all natural numbers. Denote by $S(\mathcal{M})$ the set of all measurable operators affiliated with the von Neumann algebra $\mathcal{M}$.

A linear operator $T$ affiliated with $\mathcal{M}$ is said to be locally measurable with respect to $\mathcal{M}$, if there exists a sequence $\left\{Z_{n}\right\}_{n=1}^{\infty} \subset \mathcal{P}(\mathcal{Z}(\mathcal{M}))$ such that $Z_{n} \uparrow I$ and $Z_{n} T \in S(\mathcal{M})$ for every $n \in \mathbb{N}$. Denote by $L S(\mathcal{M})$ the set of all locally measurable operators affiliated with the von Neumann algebra $\mathcal{M}$.

In [10], M. Muratov and V. Chilin prove that $S(\mathcal{M})$ and $L S(\mathcal{M})$ are both unital *-algebras and $\mathcal{M} \subset S(\mathcal{M}) \subset L S(\mathcal{M})$; the authors also show that if $\mathcal{M}$ is a finite von Neumann algebra or $\operatorname{dim}(\mathcal{Z}(\mathcal{M}))<\infty$, then $S(\mathcal{M})=L S(\mathcal{M})$; if $\mathcal{M}$ is a type III von Neumann algebra and $\operatorname{dim}(\mathcal{Z}(\mathcal{M}))=\infty$, then $S(\mathcal{M})=\mathcal{M}$ and $L S(\mathcal{M}) \neq \mathcal{M}$.

In [14], I. Segal shows that the algebraic and topological properties of the measurable operators algebra $S(\mathcal{M})$ are similar to the von Neumann algebra $\mathcal{M}$. If $\mathcal{M}$ is a commutative von Neumann algebra, then $\mathcal{M}$ is *-isomorphic to the algebra $L^{\infty}(\Omega, \Sigma, \mu)$ of all essentially bounded measurable complex functions on a measure space $(\Omega, \Sigma, \mu)$; 
and $S(\mathcal{M})$ is $*$-isomorphic to the algebra $L^{0}(\Omega, \Sigma, \mu)$ of all measurable almost everywhere finite complex-valued functions on $(\Omega, \Sigma, \mu)$. In [4], A. Ber, V. Chilin and F. Sukochev show that there exists a derivation on $L^{0}(0,1)$ is not an inner derivation, and the derivation is discontinuous in the measure topology. This result means that the properties of derivations on $S(\mathcal{M})$ are different from the derivations on $\mathcal{M}$.

So far, there are no papers on the study of the centralizable mappings on algebras of locally measurable operators. This paper is organized as follows.

In Section 2, we suppose that $\mathcal{M}$ is a von Neumann algebra and recall the definition of local measurable topology $t(\mathcal{M})$ on $L S(\mathcal{M})$.

Let $\mathcal{A}$ be a subalgebra of $L S(\mathcal{M})$. Denote by $L_{t(\mathcal{M})}(\mathcal{A}, L S(\mathcal{M}))$ the set of all continuous linear mappings with respect to the local measure topology $t(\mathcal{M})$ from $\mathcal{A}$ into $L S(\mathcal{M})$. Suppose that $G$ is a fixed element in $\mathcal{A}$, we say that $G$ is a fullcentralizable point of $L_{t(\mathcal{M})}(\mathcal{A}, L S(\mathcal{M}))$ if every continuous (with respect to the local measure topology $t(\mathcal{M})$ ) centralizable mapping at $G$ from $\mathcal{A}$ into $\mathcal{M}$ is a centralizer.

In Section 3, we show that if $\mathcal{M}$ is a von Neumann algebra without direct summands of type $\mathrm{I}_{1}$ and type $\mathrm{II}$, and $\mathcal{A}$ is a $*$-subalgebra with $\mathcal{M} \subseteq \mathcal{A} \subseteq L S(\mathcal{M})$, then every element $G$ in $\mathcal{A}$ is a full-centralizable point of $L_{t(\mathcal{M})}(\mathcal{A}, L S(\mathcal{M}))$.

\section{Preliminaries}

Let $\mathcal{H}$ be a complex Hilbert space and $\mathcal{M}$ be a von Neumann algebra on $\mathcal{H}$. Suppose that $T$ is a closed operator with a dense domain $\mathcal{D}(T)$ in $\mathcal{H}$. Let $T=U|T|$ be the polar decomposition of $T$, where $|T|=\left(T^{*} T\right)^{\frac{1}{2}}$ and $U$ is a partial isometry in $B(\mathcal{H})$. Denote by $l(T)=U U^{*}$ the left support of $T$ and by $r(T)=U^{*} U$ the right support of $T$, clearly, $l(T) \sim u(T)$. In [10], M. Muratov and V. Chilin show that $T \in S(\mathcal{M})$ (resp. $T \in L S(\mathcal{M})$ ) if and only if $|T| \in S(\mathcal{M})$ (resp. $|T| \in L S(\mathcal{M})$ ) and $U \in \mathcal{M}$.

In the following, we recall the definition of the local measure topology. Let $\mathcal{M}$ be a commutative von Neumann algebra, in [15], M. Takesaki proves that there exists a *-isomorphism from $\mathcal{M}$ onto the $*$-algebra $L^{\infty}(\Omega, \Sigma, \mu)$, where $\mu$ is a measure satisfying the direct sum property. The direct sum property means that the Boolean algebra of all projections in $L^{\infty}(\Omega, \Sigma, \mu)$ is total order, and for every non-zero projection $p$ in $\mathcal{M}$, there exists a non-zero projection $q \leq p$ with $\mu(q)<\infty$. Consider $L S(\mathcal{M})=S(\mathcal{M})=$ $L^{0}(\Omega, \Sigma, \mu)$ of all measurable almost everywhere finite complex-valued functions on $(\Omega, \Sigma, \mu)$. Define the local measure topology $t\left(L^{\infty}(\Omega)\right)$ on $L^{0}(\Omega, \Sigma, \mu)$, that is, the Hausdorff vector topology, whose base of neighborhoods of zero is given by

$$
\begin{aligned}
W(B, \varepsilon, \delta)= & \left\{f \in L^{0}(\Omega, \Sigma, \mu): \text { there exists a set } E \in \Sigma\right. \text { such that } \\
& \left.E \subset B, \mu(B \backslash E) \leq \delta, f_{\chi_{E}} \in L^{\infty}(\Omega, \Sigma, \mu),\left\|f_{\chi_{E}}\right\|_{L^{\infty}(\Omega, \Sigma, \mu)} \leq \varepsilon\right\},
\end{aligned}
$$


where $\varepsilon, \delta>0, B \in \Sigma, \mu(B)<\infty$ and $\chi_{E}(\omega)=1$ when $\omega \in E, \chi_{E}(\omega)=0$ when $\omega \notin E$. Suppose that $\left\{f_{\alpha}\right\} \subset L^{0}(\Omega, \Sigma, \mu)$ and $f \in L^{0}(\Omega, \Sigma, \mu)$, if $f_{\alpha} \chi_{B} \rightarrow f \chi_{B}$ in the measure $\mu$ for every $B \in \Sigma$ with $\mu(B)<\infty$, then we denote by $f_{\alpha} \stackrel{t\left(L^{\infty}(\Omega)\right)}{\longrightarrow} f$. In [17], Yeadon show the topology $t\left(L^{\infty}(\Omega)\right)$ dose not change if the measure $\mu$ is replaced with an equivalent measure.

If $\mathcal{M}$ is an arbitrary von Neumann algebra and $\mathcal{Z}(\mathcal{M})$ is the center of $\mathcal{M}$, then there exists a $*$-isomorphism $\varphi$ from $\mathcal{Z}(\mathcal{M})$ onto the $*$-algebra $L^{\infty}(\Omega, \Sigma, \mu)$, where $\mu$ is a measure satisfying the direct sum property. Denote by $L^{+}(\Omega, \Sigma, \mu)$ the set of all measurable real-valued positive functions on $(\Omega, \Sigma, \mu)$. In [14], Segal shows that there exists a mapping $\Delta$ from $\mathcal{P}(\mathcal{M})$ into $L^{+}(\Omega, \Sigma, \mu)$ satisfying the following conditions:

$\left(\mathbb{D}_{1}\right) \Delta(P) \in L_{+}^{0}(\Omega, \Sigma, \mu)$ if and only if $P \in \mathcal{P}_{\text {fin }}(\mathcal{M})$;

$\left(\mathbb{D}_{2}\right) \Delta(P \vee Q)=\Delta(P)+\Delta(Q)$ if $P Q=0$;

$\left(\mathbb{D}_{3}\right) \Delta\left(U^{*} U\right)=\Delta\left(U U^{*}\right)$ for every partial isometry $U \in \mathcal{M}$;

$\left(\mathbb{D}_{4}\right) \Delta(Z P)=\varphi(Z) \Delta(P)$ for every $Z \in \mathcal{P}(\mathcal{Z}(\mathcal{M}))$ and every $P \in \mathcal{P}(\mathcal{M})$;

$\left(\mathbb{D}_{5}\right)$ if $P_{\alpha}, P \in \mathcal{P}(\mathcal{M}), \alpha \in \Gamma$ and $P_{\alpha} \uparrow P$, then $\Delta(P)=\sup _{\alpha \in \Gamma} \Delta\left(P_{\alpha}\right)$.

In addition, $\Delta$ is called a dimension function on $\mathcal{P}(\mathcal{M})$ and $\Delta$ also satisfies the following two conditions:

$\left(\mathbb{D}_{6}\right)$ if $\left\{P_{n}\right\}_{n=1}^{\infty} \subset \mathcal{P}(\mathcal{M})$, then $\Delta\left(\sup _{n \geq 1} P_{n}\right) \leq \sum_{n=1}^{\infty} \Delta\left(P_{n}\right)$; moreover, if $P_{n} P_{m}=0$ when $n \neq m$, then $\Delta\left(\sup _{n \geq 1} P_{n}\right)=\sum_{n=1}^{\infty} \Delta\left(P_{n}\right)$;

$\left(\mathbb{D}_{7}\right)$ if $\left\{P_{n}\right\}_{n=1}^{\infty} \subset \mathcal{P}(\mathcal{M})$ and $P_{n} \downarrow 0$, then $\Delta\left(P_{n}\right) \rightarrow 0$ almost everywhere.

For arbitrary scalars $\varepsilon, \gamma>0$ and a set $B \in \Sigma, \mu(B)<\infty$, we let

$V(B, \varepsilon, \gamma)=\{T \in L S(\mathcal{M}):$ there exist $P \in \mathcal{P}(\mathcal{M})$ and $Z \in \mathcal{P}(Z(\mathcal{M})$ such that

$$
\left.T P \in \mathcal{M},\|T P\|_{\mathcal{M}} \leq \varepsilon, \varphi\left(Z^{\perp}\right) \in W(B, \varepsilon, \gamma) \text { and } \Delta\left(Z P^{\perp}\right) \leq \varepsilon \varphi(Z)\right\},
$$

where $\|\cdot\|_{\mathcal{M}}$ is the $C^{*}$-norm on $\mathcal{M}$. In [17], Yeadon shows that the system of sets

$$
\{T+V(B, \varepsilon, \gamma): T \in L S(\mathcal{M}), \varepsilon, \gamma>0, B \in \Sigma \text { and } \mu(B)<\infty\}
$$

defines a Hausdorff vector topology $t(\mathcal{M})$ on $L S(\mathcal{M})$ and the sets

$$
\{T+V(B, \varepsilon, \gamma), \varepsilon, \gamma>0, B \in \Sigma \text { and } \mu(B)<\infty\}
$$

form a neighborhood base of a local measurable operator $x$ in $L S(\mathcal{M})$. In [17, Yeadon also proves that $(L S(\mathcal{M}), t(\mathcal{M}))$ is a complete topological $*$-algebra, and the topology $t(\mathcal{M})$ does not depend on the choices of dimension function $\Delta$ and $*$-isomorphism $\varphi$. The topology $t(\mathcal{M})$ on $L S(\mathcal{M})$ is called the local measure topology. Moreover, if $\mathcal{M}=B(\mathcal{H})$, then $L S(\mathcal{M})=\mathcal{M}$ and the local measure topology topology $t(\mathcal{M})$ coincides with the uniform topology $\|\cdot\|_{B(\mathcal{H})}$.

The following lemma will be used repeatedly.

Lemma 2.1. [11] Suppose that $\mathcal{M}$ is a von Neumann algebra without direct summand of type II. For every $A$ in $L S(\mathcal{M})$, there exists a sequence $\left\{Z_{i}\right\}$ of mutually orthogonal 
central projections in $\mathcal{M}$ with $\sum_{i=1}^{\infty} Z_{i}=I$, such that $A=\sum_{i=1}^{\infty} Z_{i} A$ and $Z_{i} A \in \mathcal{M}$ for every $i$.

\section{Centralizable mappings on algebras of locally measurable operators}

The following theorem is the main result in this paper.

Theorem 3.1. Suppose that $\mathcal{M}$ is a von Neumann algebra without direct summands of type $\mathrm{I}_{1}$ and type II, $\mathcal{A}$ is a $*$-subalgebra of $L S(\mathcal{M})$ containing $\mathcal{M}$. Then every element $G$ in $\mathcal{A}$ is a full-centralizable point of $L_{t(\mathcal{M})}(\mathcal{A}, L S(\mathcal{M}))$.

To prove Theorem 3.1, we need the following lemmas.

Lemma 3.2. Let $\mathcal{M}$ be a von Neumann algebra and $Z$ be a central projection in $P(\mathcal{Z}(\mathcal{M}))$. Then we have that $L S(Z \mathcal{M})=Z L S(\mathcal{M})$.

Proof. Since the unit element of $L S(Z \mathcal{M})$ is $Z$, we have that

$$
L S(Z \mathcal{M})=Z L S(Z \mathcal{M}) \subseteq Z L S(\mathcal{M})
$$

Similarly, we can obtain that $L S((I-Z) \mathcal{M}) \subseteq(I-Z) L S(\mathcal{M})$. Clearly, $\mathcal{M}=Z \mathcal{M} \oplus$ $(I-Z) \mathcal{M}$, it follows that

$$
Z L S(\mathcal{M})=Z L S(Z \mathcal{M}) \oplus Z L S((I-Z) \mathcal{M})
$$

By $L S((I-Z) \mathcal{M}) \subseteq(I-Z) L S(\mathcal{M})$, we know that $Z L S((I-Z) \mathcal{M})=0$. It means that $Z L S(\mathcal{M})=Z L S(Z \mathcal{M})=L S(Z \mathcal{M})$.

In the following, we always assume that $\mathcal{M}$ is a von Neumann algebra without direct summands of type $\mathrm{I}_{1}$ and type II on a Hilbert space $\mathcal{H}, \mathcal{A}$ is a $*$-subalgebra of $L S(\mathcal{M})$ containing $\mathcal{M}$.

Lemma 3.3. Suppose that $G$ is a fixed element in $\mathcal{A}$ and $\left\{Q_{i}\right\}_{i=1}^{n}$ is a family of mutually orthogonal central projections in $\mathcal{M}$ with sum I. If $Q_{i} G$ is a full centralizable point of $L_{t(\mathcal{M})}\left(Q_{i} \mathcal{A}, Q_{i} L S(\mathcal{M})\right)$ for every $i \in \overline{1, n}$, then $G$ is a full centralizable point of $L_{t(\mathcal{M})}(\mathcal{A}, L S(\mathcal{M}))$

Proof. Let $\phi$ be in $L_{t(\mathcal{M})}(\mathcal{A}, L S(\mathcal{M}))$ centralizable at $G$.

Firstly, we show that $\phi\left(Q_{i} \mathcal{A}\right) \subseteq Q_{i} L S(\mathcal{M})$. Let $A_{i}$ be an invertible element in $Q_{i} \mathcal{M}$, and $t$ be an arbitrary nonzero element in $\mathbb{C}$. It is easy to show that

$$
\left(I-Q_{i}+t^{-1} G A_{i}^{-1}\right)\left(\left(I-Q_{i}\right) G+t A_{i}\right)=G .
$$


Thus we have that

$$
\left(I-Q_{i}+t^{-1} G A_{i}^{-1}\right) \phi\left(\left(I-Q_{i}\right) G+t A_{i}\right)=\phi(G)
$$

Considering the coefficient of $t$, since $t$ is arbitrarily chosen, we know that $\left(I-Q_{i}\right) \phi\left(A_{i}\right)=$ 0 . It follows that $\phi\left(A_{i}\right)=Q_{i} \phi\left(A_{i}\right) \in Q_{i} L S(\mathcal{M})$ for every invertible element $A_{i}$ in $Q_{i} \mathcal{M}$. Clearly, $Q_{i} \mathcal{M}$ is a von Neumann algebra and every element in $Q_{i} \mathcal{M}$ can be written into the sum of two invertible elements in $Q_{i} \mathcal{M}$. Hence we have that $\phi\left(A_{i}\right) \in Q_{i} L S(\mathcal{M})$ for every $A_{i}$ in $Q_{i} \mathcal{M}$.

For every $A_{i}$ in $Q_{i} \mathcal{A}$, by Lemma 2.1, we know that there exists a sequence $\left\{A_{i}^{n}\right\}$ in $Q_{i} \mathcal{M}$ converging to $A_{i}$ with respect to the local measure topology $t(\mathcal{M})$. By Lemma 3.1, we know that $Q_{i} L S(\mathcal{M})=L S\left(Q_{i} \mathcal{M}\right)$, since $\phi\left(A_{i}^{n}\right) \in Q_{i} L S(\mathcal{M})=L S\left(Q_{i} \mathcal{M}\right)$ and $\phi$ is continuous with respect to the local measure topology $t(\mathcal{M})$, we have that $\phi\left(A_{i}\right) \in Q_{i} L S(\mathcal{M})$ for every $A_{i}$ in $Q_{i} \mathcal{A}$.

In the following we show that $\phi$ is a centralizer from $\mathcal{A}$ into $L S(\mathcal{M})$.

Suppose that $A$ and $B$ are two elements in $\mathcal{A}$ with $A B=G$. Since $\left\{Q_{i}\right\}_{i=1}^{n}$ is a family of mutually orthogonal central projections in $\mathcal{A}$ with sum $I$, we know that there exist some elements $A_{i}, B_{i}$ and $G_{i}$ in $Q_{i} \mathcal{A}$ with $A=\sum_{i=1}^{n} A_{i}, B=\sum_{i=1}^{n} B_{i}$ and $G=\sum_{i=1}^{n} G_{i}$. Moreover, we have that $A_{i} B_{i}=G_{i}$.

Denote the restriction of $\phi$ in $Q_{i} \mathcal{A}$ by $\phi_{i}$. By $\phi\left(\mathcal{A}_{i}\right) \subseteq Q_{i} L S(\mathcal{M})$, it implies that

$$
\sum_{i=1}^{n} \phi\left(G_{i}\right)=\phi(G)=\phi(A) B=\sum_{i=1}^{n} \phi\left(A_{i}\right) \sum_{i=1}^{n} B_{i}=\sum_{i=1}^{n} \phi\left(A_{i}\right) B_{i} .
$$

Hence we can obtain that $\phi_{i}\left(G_{i}\right)=\phi_{i}\left(A_{i}\right) B_{i}$. Similarly, we have that $\phi_{i}\left(G_{i}\right)=$ $A_{i} \phi_{i}\left(B_{i}\right)$. By assumption, $G_{i}$ is a full-centralizable point of $L_{t(\mathcal{M})}\left(Q_{i} \mathcal{A}, Q_{i} L S(\mathcal{M})\right)$ for every $i \in \overline{1, n}$, that is $\phi_{i}$ is a centralizer from $Q_{i} \mathcal{A}$ into $Q_{i} L S(\mathcal{M})$ ). It follows that

$$
\phi(A)=\sum_{i=1}^{n} \phi_{i}\left(A_{i}\right)=\sum_{i=1}^{n} \phi_{i}\left(Q_{i}\right) A_{i}=\sum_{i=1}^{n} \phi_{i}\left(Q_{i}\right) \sum_{i=1}^{n} A_{i}=\phi(I) A .
$$

Similarly, we can prove $\phi(A)=A \phi(I)$. It means that $G$ is a full-centralizable point of $L_{t(\mathcal{M})}(\mathcal{A}, L S(\mathcal{M}))$.

For a unital algebra $\mathcal{A}$ and a unital left $\mathcal{A}$-module $\mathcal{M}$, we call an element $A$ in $\mathcal{A}$ a right separating point of $\mathcal{M}$ if $M A=0$ implies $M=0$ for every $M \in \mathcal{M}$. It is easy to see that every right invertible element in $\mathcal{A}$ is a right separating point of $\mathcal{M}$.

Lemma 3.4. Suppose that $G$ is a fixed element in $\mathcal{A}$. If $G$ is injective and the range of $G$ is dense in $\mathcal{H}$, then $G$ is a full-centralizable point of of $L_{t(\mathcal{M})}(\mathcal{A}, L S(\mathcal{M}))$.

Proof. Firstly, we show that $G$ is a right separating point of $L S(\mathcal{M})$. Let $A$ be in $L S(\mathcal{M})$ with $A G=0$, by Lemma [2.1, we know that there exists a sequence $\left\{Z_{i}\right\}$ 
of mutually orthogonal central projections in $\mathcal{M}$ with $\sum_{i=1}^{\infty} Z_{i}=I$, such that $A=$ $\sum_{i=1}^{\infty} Z_{i} A$ and $Z_{i} A \in \mathcal{M}$ for every $i$.

By $A G=0$, we have that $\sum_{i=1}^{\infty} Z_{i} A G=0$. Since $\left\{Z_{i}\right\}$ are mutually orthogonal projections, it follows that $Z_{i} A G=0$. By the range of $G$ is dense in $\mathcal{H}$ and $Z_{i} A \in \mathcal{M}$ for every $i$, it is easy to show that $Z_{i} A=0$ for every $i$. It means that $A=\sum_{i=1}^{\infty} Z_{i} A=0$.

Let $\phi$ be in $L_{t(\mathcal{M})}(\mathcal{A}, L S(\mathcal{M}))$ centralizable at $G$ and $A$ be an invertible element in $\mathcal{M}$. It follows that

$$
\phi(I) G=\phi(G)=\phi\left(A A^{-1} G\right)=\phi(A) A^{-1} G .
$$

Since $G$ is a right separating point of $L S(\mathcal{M})$, we have $\phi(I)=\phi(A) A^{-1}$. That is $\phi(A)=\phi(I) A$ for every invertible element $A$ in $\mathcal{M}$. It follows that $\phi(A)=\phi(I) A$ for every $A$ in $\mathcal{M}$. Since $\phi$ is continuous with respect to the local measure topology $t(\mathcal{M})$, we know that $\phi(A)=\phi(I) A$ for every $A$ in $\mathcal{A}$.

Similarly, by $\operatorname{ker}(G)=\{0\}$, we can obtain that $\phi(A)=A \phi(I)$ for every $A$ in $\mathcal{A}$. It means that $\phi$ is a centralizer from $\mathcal{A}$ into $L S(\mathcal{M})$.

Lemma 3.5. $G=0$ is a full-centralizable point of $L_{t(\mathcal{M})}(\mathcal{A}, L S(\mathcal{M}))$.

Proof. Since $\mathcal{M}$ is a von Neumann algebra without direct summand of type $\mathrm{I}_{1}$, it is well known that $\mathcal{M}$ is generated algebraically by all idempotents in $\mathcal{M}$.

Let $\phi$ be in $L_{t(\mathcal{M})}(\mathcal{A}, L S(\mathcal{M}))$ centralizable at $G$. Define a bilinear mapping $\varphi$ from $\mathcal{M} \times \mathcal{M}$ into $L S(\mathcal{M})$ by $\varphi(A, B)=\phi(A) B$ for each $A, B$ in $\mathcal{M}$. By assumption we have that $\phi(A) B=0$ for each $A, B$ in $\mathcal{M}$ with $A B=0$. It follows that $A B=0$ implies $\varphi(A, B)=0$. By [7, Theorem 4.1], we can obtain that $\varphi(A, I)=\varphi(I, A)$, it implies that $\phi(A)=\phi(I) A$ for every $A$ in $\mathcal{M}$. It also holds for every $A$ in $\mathcal{A}$, since $\phi$ is continuous with respect to the local measure topology $t(\mathcal{M})$.

Similarly, we can prove that $\phi(A)=A \phi(I)$ for every $A$ in $\mathcal{A}$. It means that $\phi$ is a centralizer from $\mathcal{A}$ into $L S(\mathcal{M})$.

Let $A$ be an element in $\mathcal{A}$. The central carrier $\mathcal{C}(A)$ of $A$ in a von Neumann algebra $\mathcal{M}$ is the projection $I-P$, where $P$ is the union of all central projections $P_{\alpha}$ in $\mathcal{Z}(\mathcal{M})$ such that $P_{\alpha} A=0$.

Lemma 3.6. Suppose that $G$ is a fixed element in $\mathcal{A}$. If $\mathcal{C}(P)=\mathcal{C}(I-P)=I$, where $P$ is the range projection of $G$, then $G$ is a full-centralizable point of $L_{t(\mathcal{M})}(\mathcal{A}, L S(\mathcal{M}))$.

Proof. Let $P_{1}=P, P_{2}=I-P$ and denote $P_{i} \mathcal{A} P_{j}$ and $P_{i} L S(\mathcal{M}) P_{j}$ by $\mathcal{A}_{i j}$ and $\mathcal{B}_{i j}$, respectively, $i, j=1,2$. For every $A$ in $\mathcal{A}$, denote $P_{i} A P_{j}$ by $A_{i j}$.

Firstly, we claim that for every element $A$ in $L S(\mathcal{M})$, the condition $A \mathcal{A}_{i j}=0$ implies $A P_{i}=0$ and $\mathcal{A}_{i j} A=0$ implies $P_{j} A=0$. 
Indeed, since $\mathcal{C}\left(P_{j}\right)=I$, by [9, Proposition 5.5.2] and $\mathcal{M} \subseteq \mathcal{A}$, we know that the range of $\mathcal{A} P_{j}$ is dense in $\mathcal{H}$. Thus $A P_{i} \mathcal{A} P_{j}=0$ implies $A P_{i}=0$. On the other hand, if $\mathcal{A}_{i j} A=0$, then $A^{*} \mathcal{A}_{j i}=0$. Hence $A^{*} P_{j}=0$ and $P_{j} A=0$.

Besides, since $P_{1}=P$ is the range projection of $G$, we have that $P_{1} G=G$. Moreover, for every element $A$ in $L S(\mathcal{M}), A G=0$ if and only if $A P_{1}=0$.

Let $\phi$ be in $L_{t(\mathcal{M})}(\mathcal{A}, L S(\mathcal{M}))$ centralizable at $G$. In the following, we show that $\phi\left(\mathcal{A}_{i j}\right) \subseteq \mathcal{B}_{i j}$, respectively, $i, j=1,2$. Suppose that $A_{11}$ is an invertible element in $\mathcal{A}_{11}$, and $A_{12}, A_{21}, A_{22}$ are arbitrary elements in $\mathcal{A}_{12}, \mathcal{A}_{21}, \mathcal{A}_{22}$, respectively. Let $t$ be an arbitrary nonzero element in $\mathbb{C}$.

Claim 1: $\phi\left(\mathcal{A}_{12}\right) \subseteq \mathcal{B}_{12}$.

By $\left(P_{1}+t A_{12}\right) G=G$, we have that $\phi(G)=\phi\left(P_{1}+t A_{12}\right) G$. It implies that $\phi\left(A_{12}\right) G=0$. Hence $\phi\left(A_{12}\right) P_{1}=0$.

By $\left(P_{1}+t A_{12}\right) G=G$, we have that $\phi(G)=\left(P_{1}+t A_{12}\right) \phi(G)$. It follows that $A_{12} \phi(G)=A_{12} \phi\left(P_{1}\right) G=0$. Thus $A_{12} \phi\left(P_{1}\right) P_{1}=0$ and $P_{2} \phi\left(P_{1}\right) P_{1}=0$.

By $\left(A_{11}+t A_{11} A_{12}\right)\left(A_{11}^{-1} G-A_{12} A_{22}+t^{-1} A_{22}\right)=G$, we have that

$$
\phi\left(A_{11}+t A_{11} A_{12}\right)\left(A_{11}^{-1} G-A_{12} A_{22}+t^{-1} A_{22}\right)=\phi(G) .
$$

Since $t$ is arbitrarily chosen in (3.1), we can obtain that

$$
\phi\left(A_{11}\right)\left(A_{11}^{-1} G-A_{12} A_{22}\right)+\phi\left(A_{11} A_{12}\right) A_{22}=\phi(G) .
$$

Since $A_{12}$ is also arbitrarily chosen, we can obtain $\phi\left(A_{11}\right) A_{12} A_{22}=\phi\left(A_{11} A_{12}\right) A_{22}$. Taking $A_{22}=P_{2}$, since $\phi\left(A_{12}\right) P_{1}=0$, we have

$$
\phi\left(A_{11} A_{12}\right)=\phi\left(A_{11}\right) A_{12}
$$

Taking $A_{11}=P_{1}$, by $P_{2} \phi\left(P_{1}\right) P_{1}=0$, it implies that

$$
P_{2} \phi\left(A_{12}\right)=P_{2} \phi\left(P_{1}\right) A_{12}=0 .
$$

Thus we can obtain that

$$
\phi\left(A_{12}\right)=\phi\left(A_{12}\right) P_{1}+P_{1} \phi\left(A_{12}\right) P_{2}+P_{2} \phi\left(A_{12}\right) P_{2}=P_{1} \phi\left(A_{12}\right) P_{2} \subseteq \mathcal{B}_{12} .
$$

Claim $2 \phi\left(\mathcal{A}_{11}\right) \subseteq \mathcal{B}_{11}$

Considering the coefficient of $t^{-1}$ in (3.1), we have that $\phi\left(A_{11}\right) A_{22}=0$. Thus $\phi\left(A_{11}\right) P_{2}=0$. By (3.2), we obtain that $P_{2} \phi\left(A_{11}\right) A_{12}=P_{2} \phi\left(A_{11} A_{12}\right)=0$. It follows that $P_{2} \phi\left(A_{11}\right) P_{1}=0$. Therefore, $\phi\left(A_{11}\right)=P_{1} \phi\left(A_{11}\right) P_{1} \subseteq \mathcal{B}_{11}$ for every invertible element $A_{11}$ in $\mathcal{A}_{11}$. Since $\phi$ is continuous with respect to the local measure topology $t(\mathcal{M})$, it implies that $\phi\left(A_{11}\right) \subseteq \mathcal{B}_{11}$ for every $A_{11}$ in $\mathcal{A}_{11}$.

Claim $3 \phi\left(\mathcal{A}_{22}\right) \subseteq \mathcal{B}_{22}$. 
By $\left(A_{11}+t A_{11} A_{12}\right)\left(A_{11}^{-1} G-A_{12} A_{22}+t^{-1} A_{22}\right)=G$, we can obtain that

$$
\left(A_{11}+t A_{11} A_{12}\right) \phi\left(A_{11}^{-1} G-A_{12} A_{22}+t^{-1} A_{22}\right)=\phi(G)
$$

Through a similar discussion for equation (3.1), we can show that

$$
P_{1} \phi\left(A_{22}\right)=0 \text { and } \phi\left(A_{12} A_{22}\right)=A_{12} \phi\left(A_{22}\right)
$$

Thus $A_{12} \phi\left(A_{22}\right) P_{1}=\phi\left(A_{12} A_{22}\right) P_{1}=0$. It follows that $P_{2} \phi\left(A_{22}\right) P_{1}=0$. Therefore, $\phi\left(A_{22}\right)=P_{2} \phi\left(A_{22}\right) P_{2} \subseteq \mathcal{B}_{22}$.

Claim $4 \phi\left(\mathcal{A}_{21}\right) \subseteq \mathcal{B}_{21}$.

By $\left(A_{11}+t A_{11} A_{12}\right)\left(A_{11}^{-1} G-A_{12} A_{21}+t^{-1} A_{21}\right)=G$, we have that

$$
\left(A_{11}+t A_{11} A_{12}\right) \phi\left(A_{11}^{-1} G-A_{12} A_{21}+t^{-1} A_{21}\right)=\phi(G) .
$$

According to this equation, we can similarly obtain that $P_{1} \phi\left(A_{21}\right)=0$ and

$$
A_{12} \phi\left(A_{21}\right)=\phi\left(A_{12} A_{21}\right)
$$

Hence $A_{12} \phi\left(A_{21}\right) P_{2}=\phi\left(A_{12} A_{21}\right) P_{2}=0$. It follows that $P_{2} \phi\left(A_{21}\right) P_{2}=0$. Therefore, $\phi\left(\mathcal{A}_{21}\right)=P_{2} \phi\left(A_{21}\right) P_{1} \subseteq \mathcal{B}_{21}$.

Claim $5 \phi\left(A_{i j}\right)=\phi\left(P_{i}\right) A_{i j}=A_{i j} \phi\left(P_{j}\right)$ for each $i, j \in\{1,2\}$.

By taking $A_{11}=P_{1}$ in (3.2), we have that $\phi\left(A_{12}\right)=\phi\left(P_{1}\right) A_{12}$. By taking $A_{22}=P_{2}$ in (3.4), we have that $\phi\left(A_{12}\right)=A_{12} \phi\left(P_{2}\right)$.

By (3.2), we have $\phi\left(A_{11}\right) A_{12}=\phi\left(A_{11} A_{12}\right)=\phi\left(P_{1}\right) A_{11} A_{12}$. It follows that $\phi\left(A_{11}\right)=$ $\phi\left(P_{1}\right) A_{11}$. On the other hand, $\phi\left(A_{11}\right) A_{12}=\phi\left(A_{11} A_{12}\right)=A_{11} A_{12} \phi\left(P_{2}\right)=A_{11} \phi\left(A_{12}\right)=$ $A_{11} \phi\left(P_{1}\right) A_{12}$. It follows that $\phi\left(A_{11}\right)=A_{11} \phi\left(P_{1}\right)$ for every invertible element $A_{11}$ and so for all elements in $\mathcal{A}_{11}$.

By (3.4) and (3.5), through a similar discussion as above, we can obtain that $\phi\left(A_{22}\right)=A_{22} \phi\left(P_{2}\right)=\phi\left(P_{2}\right) A_{22}$ and $\phi\left(A_{21}\right)=A_{21} \phi\left(P_{1}\right)=\phi\left(P_{2}\right) A_{21}$.

Now we have proved that $\phi\left(\mathcal{A}_{i j}\right) \subseteq \mathcal{B}_{i j}$ and $\phi\left(A_{i j}\right)=\phi\left(P_{i}\right) A_{i j}=A_{i j} \phi\left(P_{j}\right)$. It follows that

$$
\begin{aligned}
\phi(A) & =\phi\left(A_{11}+A_{12}+A_{21}+A_{22}\right) \\
& =\phi\left(P_{1}\right)\left(A_{11}+A_{12}+A_{21}+A_{22}\right)+\phi\left(P_{2}\right)\left(A_{11}+A_{12}+A_{21}+A_{22}\right) \\
& =\phi\left(P_{1}+P_{2}\right)\left(A_{11}+A_{12}+A_{21}+A_{22}\right) \\
& =\phi(I) A .
\end{aligned}
$$

Similarly, we can prove that $\phi(A)=A \phi(I)$.

In the following, we give the proof of our main result. 
Proof of the Theorem 3.1. Let $Q_{1}=I-\mathcal{C}(I-P), Q_{2}=I-\mathcal{C}(P)$, and $Q_{3}=I-Q_{1}-Q_{2}$, where $P$ is the range projection of $G$. Obviously, $Q_{1} \leq P$ and $Q_{2} \leq I-P$, it follows that $\left\{Q_{i}\right\}_{i=1,2,3}$ are mutually orthogonal central projections with sum $I$. Thus we have that $\mathcal{A}=\sum_{i=1}^{3}\left(Q_{i} \mathcal{A}\right)$. Denote $Q_{i} \mathcal{A}$ by $\mathcal{A}_{i}$. For every element $A$ in $\mathcal{A}$, we can write $A=\sum_{i=1}^{3} A_{i}=\sum_{i=1}^{3} Q_{i} A$

Next we divide the proof into two cases.

Case 1: Suppose that $G$ is injective, that is $\operatorname{ker}(G)=\{0\}$.

Since $Q_{1} \leq P$, we have that $\overline{r a n G_{1}}=\overline{\operatorname{ran} Q_{1} G}=Q_{1} \mathcal{H}$. By assumption we know that $G_{1}=Q_{1} G$ is injective on $Q_{1} \mathcal{H}$. By Lemma 3.4, we know that $G_{1}$ is a full-centralizable point of $L_{t(\mathcal{M})}\left(\mathcal{A}_{1}, L S\left(Q_{1} \mathcal{M}\right)\right)$.

Since $Q_{2} \leq I-P$, we have that $G_{2}=Q_{2} G=0$. By Lemma 3.5. we know that $G_{2}$ is a full-centralizable point of $L_{t(\mathcal{M})}\left(\mathcal{A}_{2}, L S\left(Q_{2} \mathcal{M}\right)\right)$.

Since $P$ is the range projection of $G$, it follows that $\overline{\operatorname{ranG} G_{3}}=\overline{\operatorname{ran} Q_{3} G}=Q_{3} P=P_{3}$. Denote the central carrier of $P_{3}$ in $\mathcal{A}_{3}$ by $\mathcal{C}_{\mathcal{A}_{3}}\left(P_{3}\right)$. We have that

$$
Q_{3}-\mathcal{C}_{\mathcal{A}_{3}}\left(P_{3}\right) \leq Q_{3}-P_{3}=Q_{3}(I-P) \leq I-P .
$$

Obviously, $Q_{3}-\mathcal{C}_{\mathcal{A}_{3}}\left(P_{3}\right)$ is a central projection orthogonal to $Q_{2}$. Thus

$$
Q_{3}-\mathcal{C}_{\mathcal{A}_{3}}\left(P_{3}\right)+I-\mathcal{C}(P) \leq I-P .
$$

It implies that $Q_{3}-\mathcal{C}_{\mathcal{A}_{3}}\left(P_{3}\right)+P \leq \mathcal{C}(P)$. Hence we have that $Q_{3}-\mathcal{C}_{\mathcal{A}_{3}}\left(P_{3}\right)=0$, that is $\mathcal{C}_{\mathcal{A}_{3}}\left(P_{3}\right)=Q_{3}$. Similarly, we can show that $\mathcal{C}_{\mathcal{A}_{3}}\left(Q_{3}-P_{3}\right)=Q_{3}$. By Lemma 3.6, we know that $G_{3}$ is a full-centralizable point of $L_{t(\mathcal{M})}\left(\mathcal{A}_{3}, L S\left(Q_{3} \mathcal{M}\right)\right)$.

By Lemma 3.2, we can obtain that $Q_{i} L S(\mathcal{M})=L S\left(Q_{i} \mathcal{M}\right)$. Hence $G_{i}$ is a fullcentralizable point of $L_{t(\mathcal{M})}\left(\mathcal{A}_{i}, Q_{i} L S(\mathcal{M})\right)$ for each $i=1,2,3$.

By Lemma 3.3, it follows that $G$ is a full-centralizable point of $L_{t(\mathcal{M})}(\mathcal{A}, L S(\mathcal{M}))$.

Case 2: Suppose that $\operatorname{ker}(G) \neq\{0\}$.

In this case, $G_{2}$ and $G_{3}$ are still full-centralizable points of $L_{t(\mathcal{M})}\left(\mathcal{A}_{2}, L S\left(Q_{2} \mathcal{M}\right)\right)$ and $L_{t(\mathcal{M})}\left(\mathcal{A}_{3}, L S\left(Q_{3} \mathcal{M}\right)\right)$, respectively.

Since $\overline{\operatorname{ran} G_{1}}=Q_{1} \mathcal{H}$, we have that $\operatorname{ker}\left(G_{1}^{*}\right)=\{0\}$. By Case 1, we know that $G_{1}^{*}$ is a full-centralizable point of $L_{t(\mathcal{M})}\left(\mathcal{A}_{1}, L S\left(Q_{1} \mathcal{M}\right)\right)$. Next we show that $G_{1}$ is also a full-centralizable point of $L_{t(\mathcal{M})}\left(\mathcal{A}_{1}, L S\left(Q_{1} \mathcal{M}\right)\right)$.

In fact, let $\phi_{1}$ be in $L_{t(\mathcal{M})}\left(\mathcal{A}_{1}, L S\left(Q_{1} \mathcal{M}\right)\right)$ centralizable at $G_{1}$. Define a linear mapping $\widetilde{\phi_{1}}$ from $\mathcal{A}_{1}$ into $L S\left(Q_{1} \mathcal{M}\right)$ by $\widetilde{\phi_{1}}(A)=\left(\phi_{1}\left(A^{*}\right)\right)^{*}$ for every $A$ in $\mathcal{A}_{1}$. Suppose that $A$ and $B$ are two elements in $\mathcal{A}_{1}$ with $A B=G_{1}$, we have that $B^{*} A^{*}=G_{1}{ }^{*}$. It follows that

$$
\phi_{1}(G)=A \phi_{1}(B)=\phi_{1}(A) B .
$$

By the definition of $\widetilde{\phi_{1}}$, we can obtain that

$$
\widetilde{\phi_{1}}\left(G^{*}\right)=B^{*} \widetilde{\phi_{1}}\left(A^{*}\right)=\widetilde{\phi_{1}}\left(B^{*}\right) A^{*} .
$$


Since $G^{*}$ is a full-centralizable point of $L_{t(\mathcal{M})}\left(\mathcal{A}_{1}, L S\left(Q_{1} \mathcal{M}\right)\right)$, we have that $\widetilde{\phi_{1}}$ is a centralizer. Thus $\phi_{1}$ is also a centralizer. It means that $G_{1}$ is a full-centralizable point of $L_{t(\mathcal{M})}\left(\mathcal{A}_{1}, L S\left(Q_{1} \mathcal{M}\right)\right)$.

By Lemma 3.3, we know $G$ is a full-centralizable point of $L_{t(\mathcal{M})}(\mathcal{A}, L S(Q \mathcal{M}))$.

\section{References}

[1] S. Albeverio, S. Ayupov, K. Kudaybergenov. Derivations on the algebra of measurable operators affiliated with a type I von Neumann algebra. Siberian Adv. Math., 2008, 18: 86-94.

[2] S. Albeverio, S. Ayupov, K. Kudaybergenov. Structure of derivations on various algebras of measurable operators for type I von Neumann algebras. J. Func. Anal., 2009, 256: 2917-2943.

[3] G. An, J.Li. Characterizations of linear mappings through zero products or zero Jordan products. Electron. J. Linear Algebra, 2016, 31: 408-424.

[4] A. Ber, V. Chilin, F. Sukochev. Non-trivial derivation on commutative regular algebras. Extracta Math., 2006, 21: 107-147.

[5] M. Brešar. Characterizing homomorphisms, derivations and multipliers in rings with idempotents. Proc. Roy. Soc. Edinburgh, Sect. A, 2007, 137: 9-21.

[6] M. Brešar, E. Kissin, S. Shulman. Lie ideals: from pure algebra to $\mathrm{C}^{*}$-algebras. J. reine angew. Math., 2008, 623: 73-121.

[7] M. Brešar. Multiplication algebra and maps determined by zero products. Linear Multilinear Algebra, 2012, 60: 763-768.

[8] J. He, J. Li, W. Qian. Characterizations of centralizers and derivations on some algebras. J. Korean Math. Soc., 2017, 54: 685-696.

[9] R. Kadison, J. Ringrose. Fundamentals of the Theory of Operator Algebras. Academic Press Inc, 1983.

[10] M. Muratov, V. Chilin. Algebras of measurable and locally measurable operators. Kyiv, Pratse In-ty matematiki NAN ukraini, 2007, 69: 390, (Russian).

[11] M. Muratov, V. Chilin. Central extensions of *-algebras of measurable operators. Reports of the National Academy of Science of Ukraine, 2009, 7: 24-28, (Russian).

[12] X. Qi, J. Hou. Characterizing centralizers and generalized derivations on triangular algebras by acting on zero product. Acta Math. Sinica (Engl. Ser.) 2013, 29: 12451256.

[13] X. Qi. Characterization of centralizers on rings and operator algebras. Acta Math. Sinica, 2013, 56: 459-468. 
[14] I. Segal. A non-commutative extension of abstract integration. Ann, Math., 1953, 57: 401-457.

[15] M. Takesaki. Theory of operator algebras I, New York, Springer-Verlag, 1979.

[16] W. Xu, R. An, J. Hou. Equivalent characterization of centralizers on B(H). Acta Mathematica Sinica, 2016, 32: 1113-1120.

[17] F. Yeadon. Convergence of measurable operators. Proc. Camb. Phil. Soc., 1973, 74: $257-268$. 